\title{
Comparison between the Effect of Hyaluronic Acid and Mesenchymal Stem Cell Therapy on Cornea of Adult Albino Rats after Exposure to Alkali Burn (Light and Electron Microscopic Study)
}

\author{
Saadia A. Shalaby, Essam M. Eid, Ali M. Ali, Samia M. Manawy, Samar F. Gad
}

\begin{abstract}
Department of anatomy and embryology. Benha faculty of medicine, Benha University, Egypt

Correspondence to: Samar F. Gad, Department of anatomy and embryology. Benha faculty of medicine, Benha University, Egypt
\end{abstract}

Email:

samar.fawzy2009@yahoo.com

Received: 26 December 2019

Accepted: 23 February 2020

\begin{abstract}
:
Background: Corneal epithelial injuries are common in both physician and veterinary ophthalmology that often causes extensive damage and results in permanent visual impairment. Aim of work: to evaluate the beneficial effect of hyaluronic acid and MSCs in treatment of experimental alkali corneal burn. Subjects and methods: thirty adult albino rats of both sexes. Ten rats were used to harvest BM-MSCs while the others were divided into four groups. Group I was the control group. Group II with unilateral alkali-burnt cornea these rats were sacrificed after 1 day and the other sacrificed after 2 weeks. Group III were rats with unilateral hyaluronic acidtreated alkali-burnt corneas for 2 weeks. Group IV were rats with MSCs treated alkali-burnt corneas. Immune histochemical staining for CD44 and vimentin was performed. The corneas were examined using light microscopic, transmission electron microscopic and morphometric studies. Results: Corneal alkali burn resulted in desquamation of corneal epithelium in group II. The epithelial cell layers had vacuolated cytoplasm with pyknotic nuclei. The stroma contained irregularly arranged collagen fibers with wide spacing and congested blood vessels with cellular infiltration. Groups III and IV showed improvement of the histological and electron microscopic
\end{abstract} changes described in group II. Conclusions: The use of MSCs in the acute stage of corneal chemical trauma was associated with faster recovery of the wounded cornea when compared with the effect of hyaluronic acid sodium salt.

Keywords: cornea, alkali burn, MSCs, hyaluronic acid 
Abbreviations: HA: Hyaluronic acid, BM-MSCs: Bone Marrow derived Mesenchymal Stem Cells, PBS: Phosphate Buffer Saline, H\&E: Haematoxylin and Eosin, SD: Standard Deviation, ANOVA: One-way Analysis of Variance, EM: Electron Microscope.

\section{Introduction}

Cornea is a transparent avascular tissue that provides a clear vision by refracting light onto the lens. Cornea acts as a barrier of the eye against infections, abrasions, and structural damage ${ }^{(1)}$.

Alkali burn corneal wounds cause reduced transparency of the cornea and may cause permanent visual impairment or even blindness. Moreover, they do not heal properly spontaneously and there is a lack of satisfactory therapy ${ }^{(1)}$.

HA is a viscoelastic glycosaminoglycan composed of alternating $\beta$-1,4-glucuronic acid and $\beta-1,3-\mathrm{N}$-acetylglucosamine. HA interacts with several cell surface receptors and provides anti-inflammatory and antiapoptotic signals to corneal cells exposed to Ultraviolent radiation ${ }^{(2)}$.MSC are promising approaches in regenerative therapies. MSC have been used in a recent technique for wound repair, regeneration, and tissue engineering procedures because the isolated cells expand rapidly and differentiate into different cell types ${ }^{(3)}$.

The aim of this study was to compare the therapeutic effects of HA and MSCs in the treatment of alkali-induced epithelial corneal defects.

\section{Material and methods}

This study was conducted on 30 albino rats of both sexes weighing between 220 and 250 $\mathrm{mg}$, taken from and housed at the laboratory animal house unit of Kasr Al-Ainy Faculty of Medicine, Cairo University (from July 2018 to November 2018). Strict maintenance and cleaning measures were applied to keep the animals in healthy state. All ethical rules for animal treatment were followed.

\section{Experimental Design}

Bone marrow was harvested by flushing the tibiae and femurs of ten rats for BM-derived MSCs culture. The other animals were divided into: Group I (4 rats); the control group was sham operated. Group II (8 rats); corneal alkali burn was created. These rats were anesthetized with intramuscular injection of $0.5 \mathrm{mg} / \mathrm{kg}$ ketamine. The right eyes of each rat were gently opened wide. Filter paper soaked in $1 \% \mathrm{NaOH}$ then placed on the cornea for 40 seconds. The cornea was then rinsed with $60 \mathrm{ml}$ of saline for 1 minute ${ }^{(2)}$. Four of these rats (subgroup IIa) were sacrificed at 1 day after alkali burn; other four rats (subgroup Ilb) were sacrificed after two weeks. 
Group III (4 rats); corneal alkali burn was done as in group II then HA sodium salt (polyfresh artificial tear) administered topically as eye drops, which was given every $2 \mathrm{~h}$, six times a day, daily starting 1 day after corneal alkali burn. These rats were sacrificed after 2 weeks. Group IV (4 rats); underwent corneal alkali burn as in group II then treated with intravenous injection of PBS containing $2 \times 106 \mathrm{MSCs}$; sacrificed at 2 weeks.

\section{Isolation of BM-derived MSCs: Bone} marrow was harvested with Dulbecco's modified Eagle's medium (DMEM,GIBCO/ BRL) supplemented with $10 \%$ fetal bovine medium(GIBCO/BRL). Cells were isolated with a density gradient [Ficoll/Paque (Pharmacia)] and re-suspended in complete culture medium supplemented with $1 \%$ penicillin- streptomycin (GIBCO/BRL). Cells were incubated at $37 \mathrm{oC}$ ' in $5 \%$ humidified Co2 for $12 \sim 14$ days as primary culture or

upon formation of large colonies. The cultures were washed and cells were trypsinized with $0.25 \%$ trypsin in $1 \mathrm{mM}$ EDTA (GIBCO/BRL). After centrifugation, cells were re-suspended and incubated in flask Falcon. The resulting cultures were referred to as first-passage cultures ${ }^{(4)}$. The MSCs were recognized in culture by inverted microscope as spindle shaped cells (fig. 1a).

\section{Labeling of stem cells with PKH26 dye:}

MSCs were labeled with the PKH26 fluorescent dye. PKH26 was purchased from Sigma Company (Saint Louis, Missouri, USA) ${ }^{(5)}$. Cornea was examined using fluorescence microscope (Leica Microsystems CMS GmbH, Wetzlar, Germany) at Kasr AlAiny Faculty of Medicine, Cairo University. Several MSCs labeled with PKH26 fluorescent dye were detected housed in the cornea by their strong red fluorescence (fig. 1 b

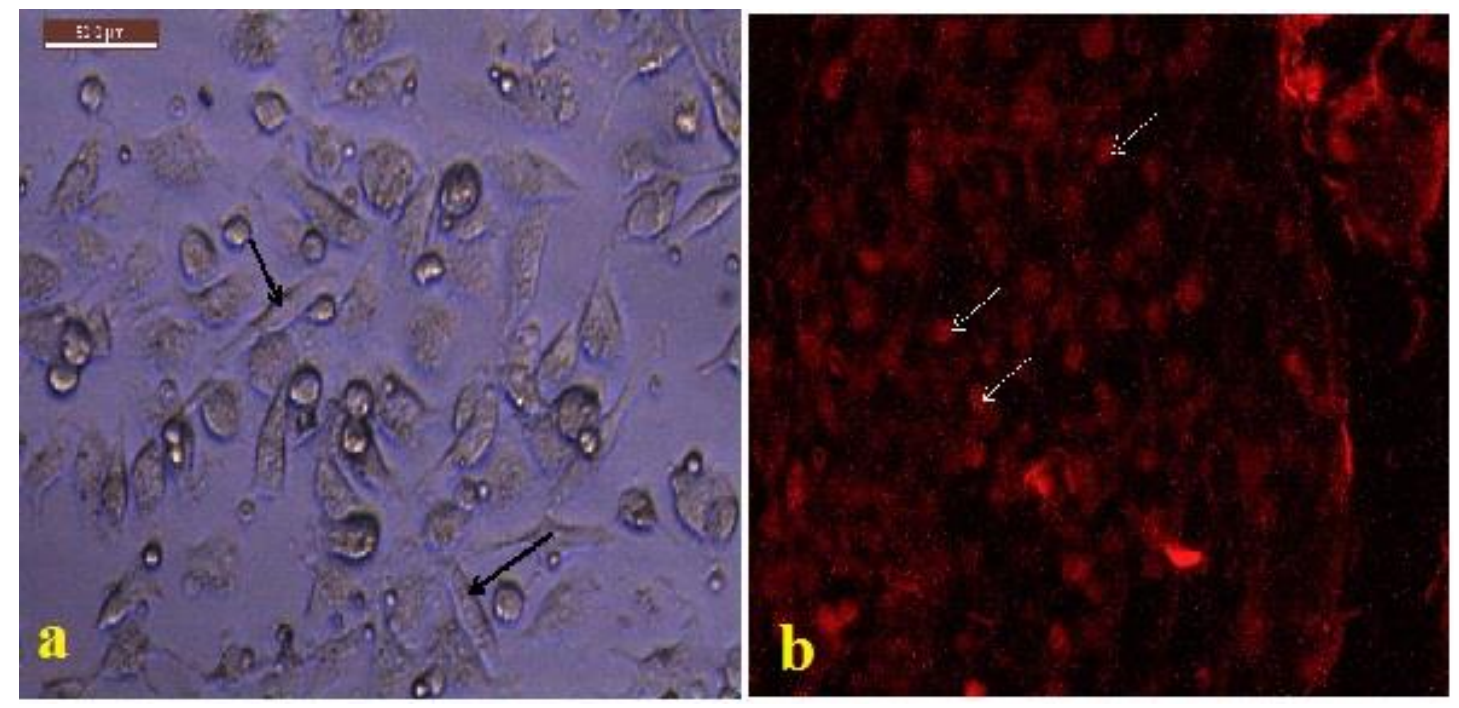

Fig. 1: (a) A photomicrograph of MSCs in culture at one week; adhesive spindle shaped cells (black arrows) (Inverted microscopic $\times$ 200). (b) A photomicrograph showing several immunostained mesenchymal stem cells (PKH 26, red fluorescent) housed in the cornea (white arrows) (Immunofluorescence, $\times \mathbf{2 0 0}$ ). 
Histological study: Specimens were fixed immediately in $10 \%$ buffered formal saline for:

- Light microscopic study: $5 \mu \mathrm{m}$ sections were cut and stained with hematoxylin and eosin $(H \& E)$ for histological study ${ }^{(6)}$.

\section{- Transmission electron microscopic study:} the corneas were collected and ultrathin sections were performed ${ }^{(7)}$. It was done and photographed in Tanta EM Unit, Faculty of Medicine, Tanta University using JOEL (JEM-100 SX, Akishima, Tokyo, Japan).

\section{Immunohistochemical study:}

Immunohistochemistry of paraffin-embedded corneal sections was performed with CD44 and vimentin antibodies by using the avidinbiotin peroxidase complex technique ${ }^{(8)}$. The slides were studied by light microscopy in Anatomy Department, Benha faculty of Medicine, Benha University.

Morphometric study: H\&E slides were assessed to measure the corneal epithelial thickness $(\mu \mathrm{m})$, stromal thickness $(\mu \mathrm{m})$ and Descemet's membrane thickness $(\mu \mathrm{m})$ at a magnification of $\times 400$. Data were expressed as group mean value $\pm \mathrm{SD}$. ANOVA with post hoc Scheffe's test was used to contrast differences between the groups. A value of $\mathrm{P}$ $<0.05$ was accepted as statistically significant.

\section{Results}

\section{H\&E results}

Group I: the rat cornea consists of nonkeratinized stratified squamous epithelium which is formed of columnar basal cells with oval nuclei, intermediate layers of polygonal cells with rounded nuclei and superficial flattened squamous cells with flattened nuclei. Bowman's layer, where the columnar cells are resting on, is ill defined seen. The corneal stroma is the thickest layer of the cornea. It appears avascular. It is formed of regularly organized collagen fibers with spindle-shaped stromal cells (Keratocytes) in between. Descemet's membrane is seen as a homogenous acidophilic noncellular layer interposed between the stroma and the underlying endothelial cells with their flat nuclei (Fig.2a)

Group II: Corneal sections for subgroup IIa show desquamation of surface and intermediate cells exposing the basal epithelial cells. Corneal stroma had disturbed collagen fibers with spaces began to appear in between fibers (Fig.2b).

Corneal sections for subgroup IIb show variability in the thickness of the corneal epithelium and disfigurement. Some of epithelial cells have vacuolated cytoplasm. Corneal stroma contained collagen fibers with wide spaces. Blood vessels are surrounded 
with inflammatory cells, Descemet's membrane cellular infiltrates (Fig.2c).

Group III: Rat corneal sections show multilayered, organized epithelium. Apparently flat cells overlap the superficial epithelial layer. The collagen fibers in the stroma are regularly arranged in posterior portion of cornea and disturbed in the anterior part with wide spaces are still present.
Descemet's membrane appeared with focal loss of some endothelial cells (Fig.2d).
Group IV: Rat corneal sections show that the layers of the cornea can be easily identified. The corneal epithelium appears continuous with very thin intact Bowman's membrane. The corneal stroma is formed of regularly arranged collagen fibers. Descemet's membrane and endothelium are apparently

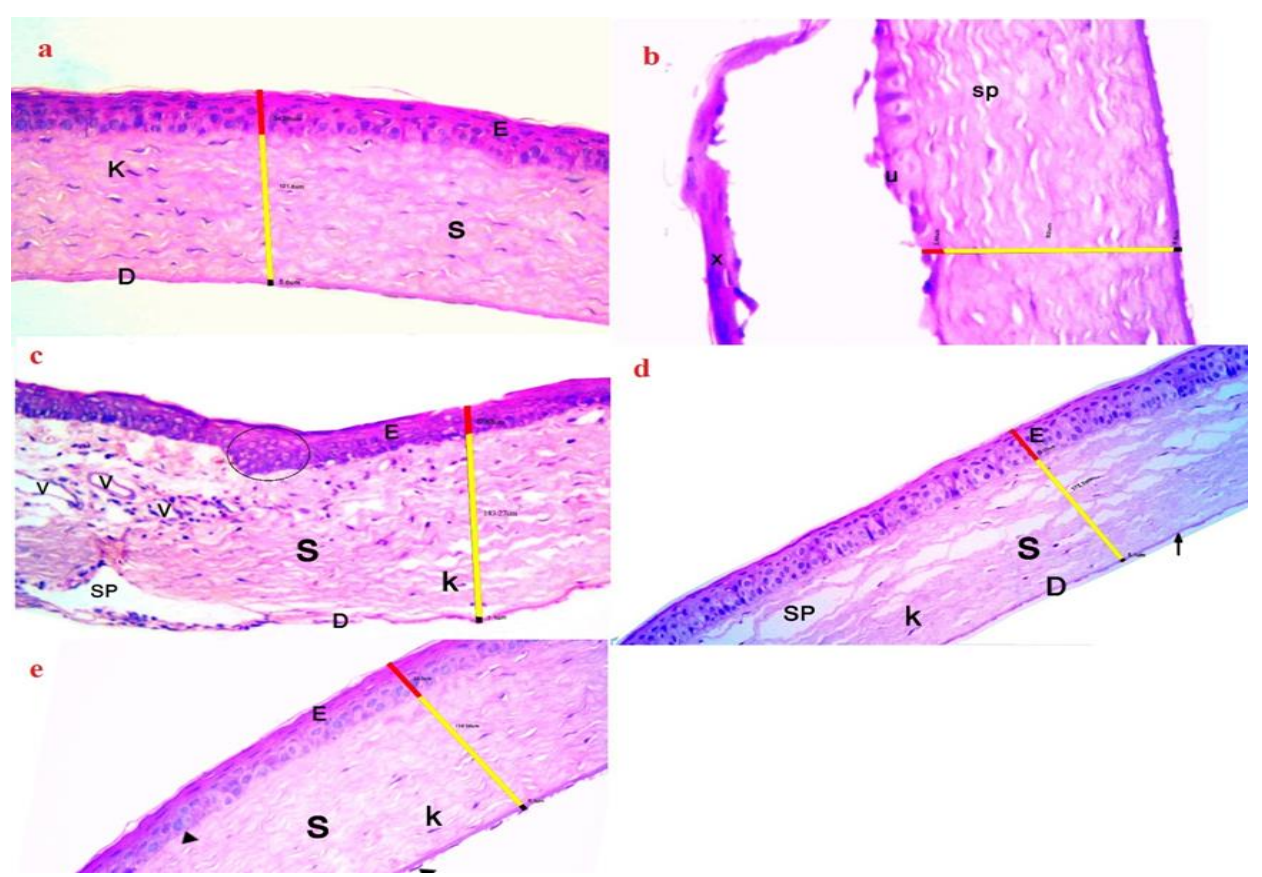

Fig. 2: (a) A photomicrograph of a section of Group I, showing non keratinized stratified squamous corneal epithelium (E); thick stroma (S) containing scattered keratocytes (K). Descemet's membrane (D) with a continuous simple squamous corneal endothelium is seen $(\mathbf{H \& E}, \times \mathbf{4 0 0})$. (b) A photomicrograph of a section of subgroup IIa, showing desquamation of surface and intermediate epithelial cells (x) exposing the intermediate and basal cells (u) of the corneal epithelium. Spacing (sp) between stromal collagen fibers is observed $(\mathbf{H} \& \mathbf{E}, \times \mathbf{4 0 0})$. (c) A photomicrograph of a section of subgroup IIb, showing disorganized corneal epithelial layer (E) with, some of the cells have vacuolated cytoplasm (circle). Wide spaces (sp) are observed between disorganized collagen fibers of stroma (S). The corneal stroma appears to be cellularly infiltrated around blood vessels (v). Descemet's membrane (D) appears to be cellularly infiltrated (H\&E, $\times$ 400). (d) A photomicrograph of a section of subgroup III, showing continuous corneal epithelium (E) with apparently flat cells overlapping the superficial epithelial layer. Bowman's membrane is very thin (head arrow).Wide spacing (SP) between collagen fibers of the stroma (S) is observed with keratocytes (K) in between. Descemet's membrane (D) appears with focal loss of some endothelial cells (arrow) (H\&E, $\times$ 400). (e) A photomicrograph of a section of group IV, showing non-keratinized stratified squamous corneal epithelium (E) comparable to that of the control group thin intact Bowman's membrane (head arrow). Collagen fibers of the stroma (S) show an apparently normal pattern with scattered keratocytes $(\mathrm{k})$. Descemet's membrane (arrow) is lined by normal endothelium (H\&E, $\mathbf{x}$ 400). 


\section{EM results}

Group I: The top layer of corneal epithelium is formed of flat cells with apical microvilli. The nucleus is flattened with a fine, light and scattered condensed chromatin granulation. The numerous electron-dense desmosomes are present at cell membranes connecting cells together (Fig.3a).

The basal layer is formed of columnar cells having spherical nucleus with fine granular chromatin. They are attached to the straight basement membrane by well-apparent hemidesmosomal junctions. Columnar cells are connected with their neighbors by desmosomes (Fig.3b).

The collagen fibers of corneal stroma within each lamella are arranged parallel to one another arranged in longitudinal and transverse planes. In between fibers, there is long, spindle shaped keratocyte with thin and long cytoplasmic processes. Its nucleus occupies the most central area with thin rim of heterochromatin at the periphery. The cell has many stacks of rough endoplasmic reticulum and mitochondria within the cytoplasm (Fig.3c).

Descemet's membrane appears as a thick homogenous electron-dense non-cellular membrane. The endothelial cell is seen as single layer of flat cell with flat nucleus which has a moderate electron-dense chromatin. Its cytoplasm shows pinocytotic vesicle

(Fig.3d)

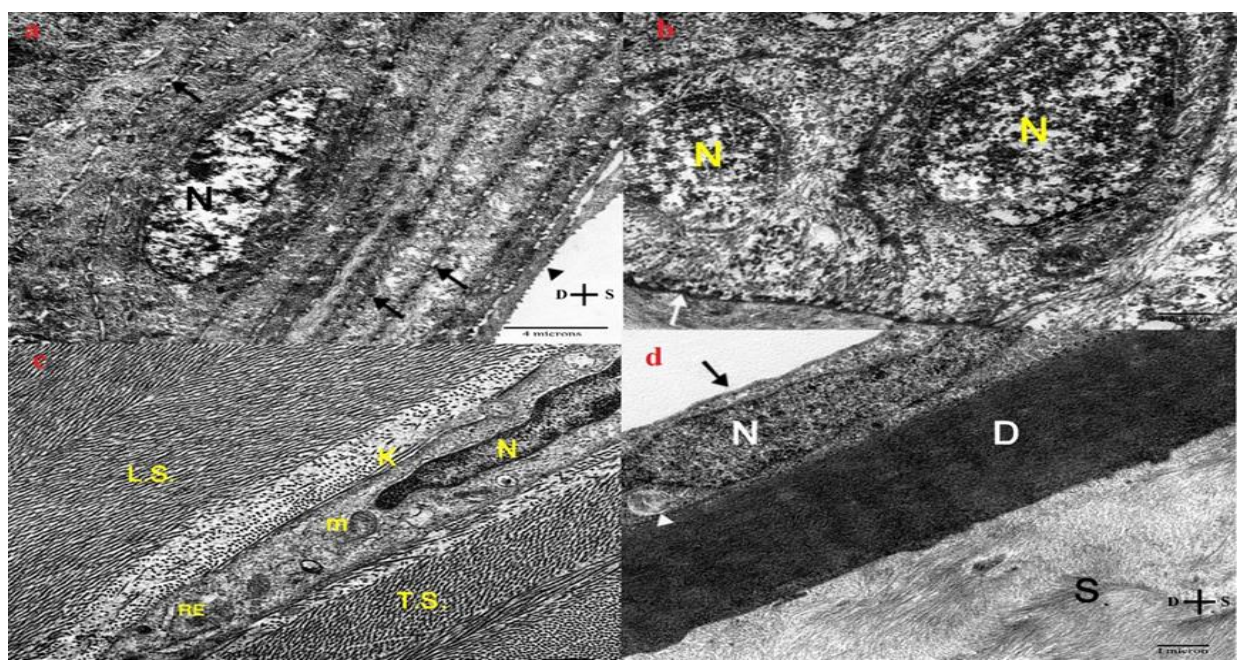

Fig. 3: (a) An electron micrograph of a section of Group I, showing superficial squamous cell with flat nucleus (N) and minute microvilli (head arrow) on the free surface. Notice the numerous electron-dense desmosomes (arrow) $(\mathbf{E M}, \times \mathbf{8 0 0 0})$. (b) An electron micrograph of a section of Group I, showing columnar cells of the basement membrane of the corneal epithelium with their round nuclei $(\mathrm{N})$. Notice regular basal lamina and hemidesmosomes appear as dotted electron-dense spots (arrow) $(\mathbf{E M}, \times \mathbf{1 7 5 0 0})$. (c) An electron micrograph of a section of Group I, showing, collagen fibers arranged in longitudinal (L.S) and transverse (T.S) planes. Notice keratocytes $(\mathrm{K})$ among the collagen fiber shows rough endoplasmic reticulum (RE) and mitochondria (m) in its cytoplasm (EM, $\times$ 17500). (d) An electron micrograph of a section of Group I, showing, homogenous electron dense Descemet's membrane (D) between the stroma (S) and the underlying endothelium (arrow). Notice flat moderate electron dense nucleus of endothelium (N) and pinocytotic vesicles (head arrow) in its cytoplasm $(\mathbf{E M}, \times \mathbf{8 0 0 0})$. 
Subgroup IIa: superficial layers of the corneal epithelium show that epithelial cells are separated from each other by wide intercellular spaces with loss of desmosomes in between. These cells have marked and heavily stained chromatin patches in their nuclei with irregularity in their shape. Wide intercellular spaces have showed mononuclear cell infiltration in between the cells (Fig.4a).

The cells of the basal layer have irregular contour with loss of their columnar shape. Some nuclei of these cells have condensed chromatin and other with pale chromatin. The basement membrane appears irregular and ill defined. Wide intercellular spaces are also noticed (Fig.4b).

The stromal keratocytes show darkly stained nuclei and many vacuoles in its cytoplasm with short cytoplasmic processes (Fig.4c).

Descemet's membrane appears regular and homogenous; however, the endothelial cells are swollen with large cytoplasmic. The endothelial cell has elongated nucleus with light stained chromatin (Fig.4d).

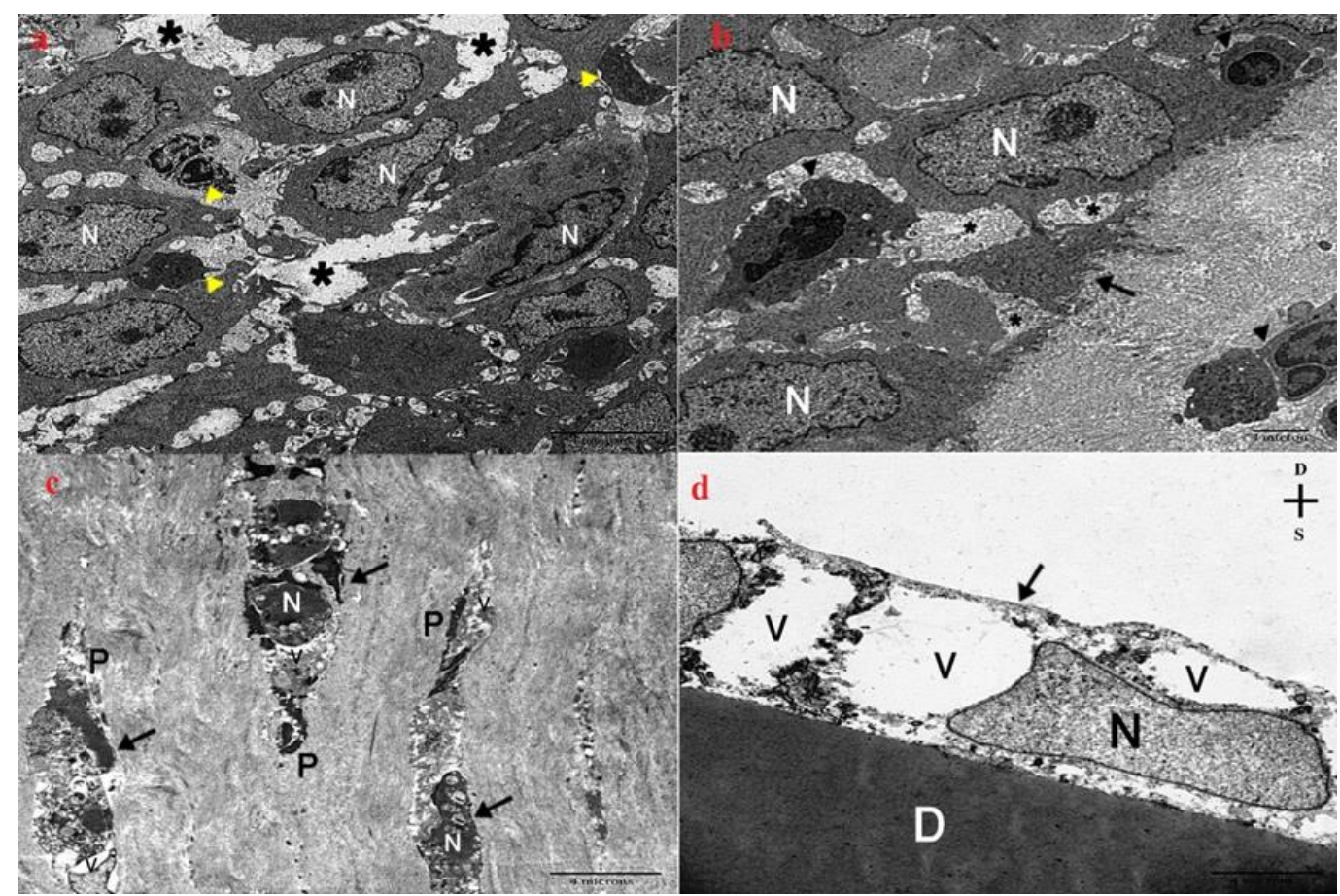

Fig. 4: (a) An electron micrograph of a section of subgroup IIa, Showing cells of the middle layer of the corneal epithelium with disfigurement in the appearance on theier nuclei $(\mathrm{N})$. Wide intercellular spaces (star) with mononuclear cellular infiltrations (head arrow) are noticed $(\mathbf{E M}, \times \mathbf{8 0 0 0})$. (b) An electron micrograph of a section of subgroup IIa, irregular contour of corneal epithelial cells with some nuclei $(\mathrm{N})$ have condensed chromatin and other with pale chromatin. The basement membrane (arrow) appears irregular with absence of hemidesmosomes. Corneal epithelium and Stroma show mononuclear cellular infiltrations (head arrow). Notice Wide intercellular spaces $($ star $)(\mathbf{E M}, \times \mathbf{8 0 0 0})$. (c) An electron micrograph of a section of subgroup IIa, Showing Keratocytes (arrow) with degenerated nuclei (N), vacuolated cytoplasm (v) and short cytoplasmic process (p) $(\mathbf{E M}, \times \mathbf{8 0 0 0})$. (d) An electron micrograph of a section of subgroup IIa, part of Descemet's membrane (D) and swollen endothelial cell (arrow). The endothelial cell has cytoplasmic vacuoles $(\mathrm{V})$, and pale irregular nucleus $(\mathrm{N})(\mathbf{E M}, \times \mathbf{8 0 0 0})$. 
Subgroup IIb: it shows loss of microvilli on the free surface of the superficial layer of the corneal epithelium. Their nuclei have light stained chromatin. Wide intercellular spaces present between them. Mononuclear cell infiltrations are obvious in the superficial layer of cornea (Fig.5a).

Cells of the basal layer appear irregular in shape containing mitochondria with partial lysis of their cristae. Their nuclei have heavily stained chromatin patch. They rest on an irregular basement membrane. They show loss of desmosomes with narrow intercellular spaces (Fig.5b).
Corneal stroma shows an irregular arrangement of the collagen fibers. Keratocyte has shrunken nuclei with condensed chromatin with little vacuolated cytoplasm and degenerated cytoplasmic processes (Fig.5c). Congested blood vessel could be seen (Fig.5d).

Descemet's membrane is lightly stained and lined with endothelial cell which has cytoplasmic vacuoles and many swollen degenerated mitochondria (Fig.5e).

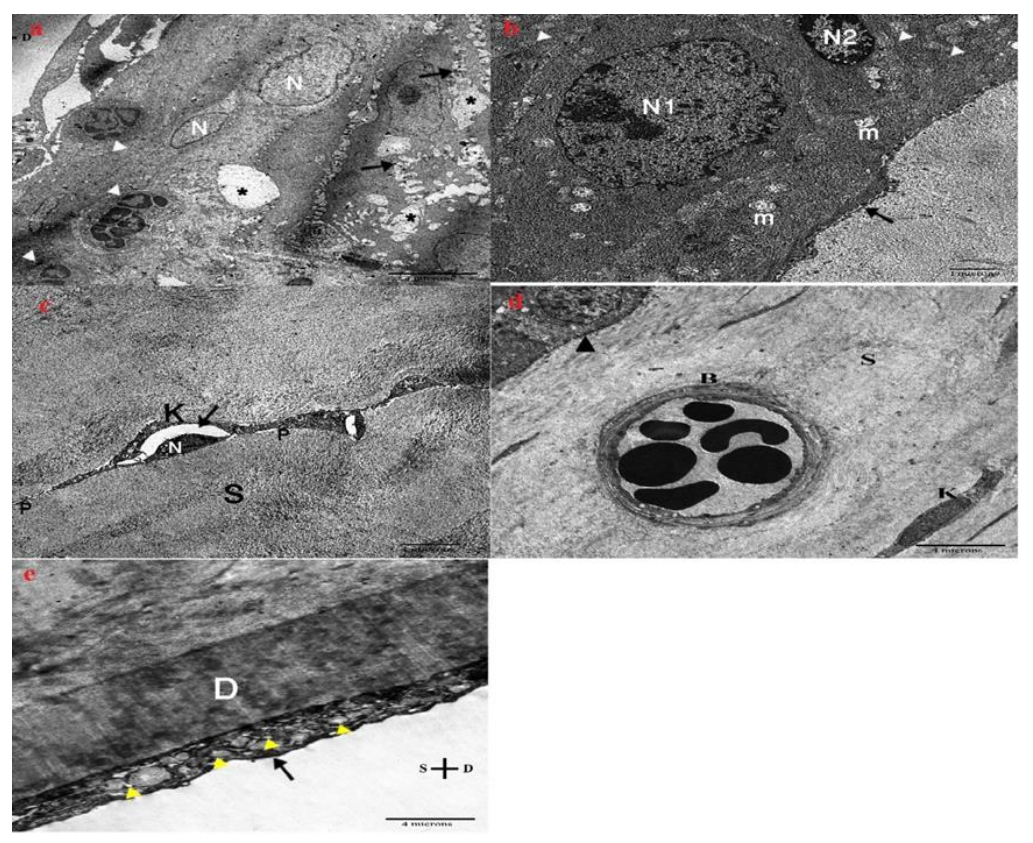

Fig. 5: (a) An electron micrograph of a section of subgroup IIb, showing cells of the superficial layer of the corneal epithelium with flat nuclei $(\mathrm{N})$. They are separated by intercellular spaces (star) due to disrupted desmosomes (arrow). Notice mononuclear cellular infiltration (head arrow) $(\mathbf{E M}, \times \mathbf{8 0 0 0})$. (b) An electron micrograph of a section of subgroup IIb, showing irregular contour of a part of columnar cell of basal layer of the corneal epithelium.one of them has dark nucleus (N1) and another one has shrunken nucleus (N2). Many mitochondria are vacuolated (head arrow) and other with partial lysis of their cristia (m). Notice an irregular basement membrane (arrow) $(\mathbf{E M}, \times \mathbf{8 0 0 0})$. (c) An electron micrograph of a section of subgroup IIb, showing keratocyte $(\mathrm{K})$ with vacuolated cytoplasm (arrow), shrunken nucleus $(\mathrm{N})$ and degenerated cytoplasmic processes (p) with vacuoles between irregular fibers of corneal stroma $(\mathrm{S})(\mathbf{E M}, \times \mathbf{8 0 0 0})$. (d) An electron micrograph of a section of subgroup IIb, showing the corneal stroma $(\mathrm{S})$ contains blood vessel (B) filled with blood cells. Notice basement membrane of corneal epithelium (head arrow) $(\mathbf{E M}, \times \mathbf{8 0 0 0})$. (e) An electron micrograph of a section of subgroup IIb, showing pale Descemet's membrane (D) and endothelial cell (arrow) with many variable sized vacuoles (head arrow) is observed $(\mathbf{E M}, \times \mathbf{8 0 0 0})$. 
Group III: it reveals flat cell with a relatively homogeneous chromatin material in the nucleus. The free surface shows irregularity with numerous apical microvilli. They are connected by desmosomes with wide intercellular gaps (Fig.6a).

The basal cells are columnar in shape with fine granular chromatin of their nucleus and prominent nucleolus connected together with desmosomes. Regular basement membrane is noticed (Fig.6b).
The collagen fibers of stroma are regular with normal Keratocytes with rough endoplasmic reticulum in its cytoplasm in between (Fig.6c).

The Descemet's membrane is homogenous moderate electron dense membrane and the endothelial cell has nucleus with irregular electron dense chromatin and multiple cytoplasmic vacuoles (Fig.6d).

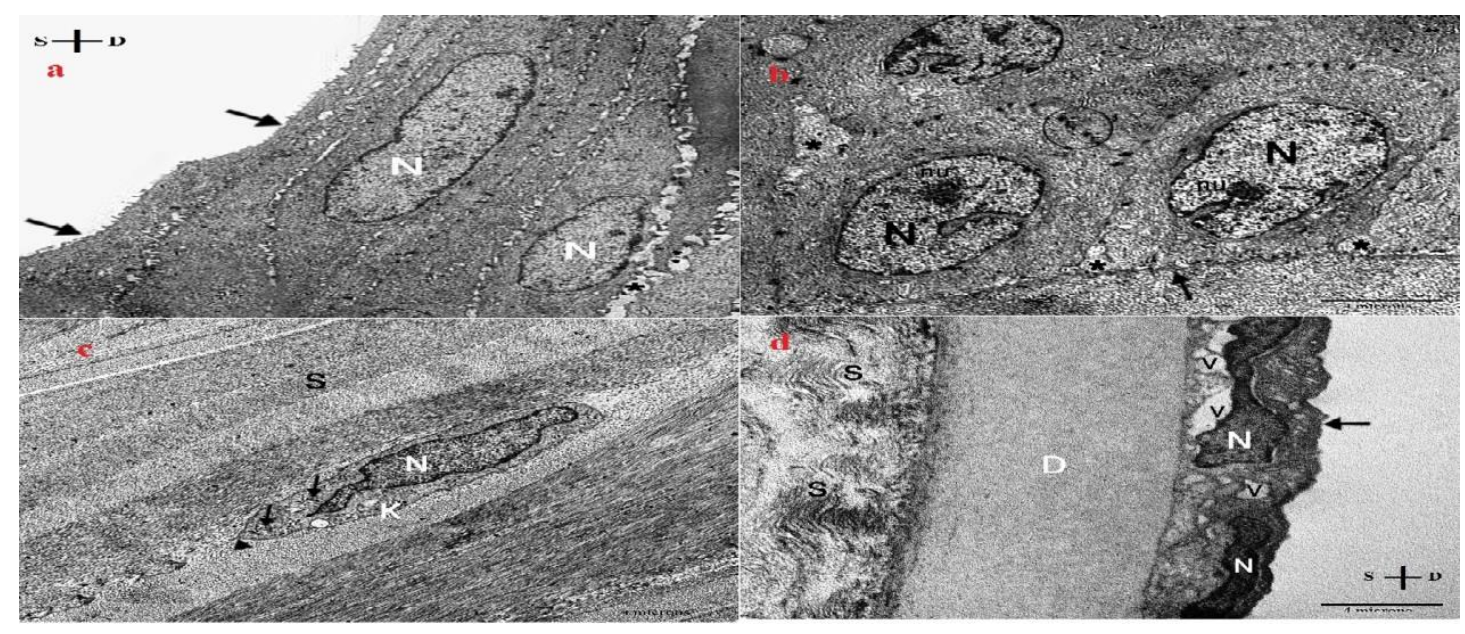

Fig. 6: (a) An electron micrograph of a section group III, showing squamous superficial corneal epithelial cells with elongated nucleus $(\mathrm{N})$ and minute microvilli (arrow) arising from superficial surface. The free surface is irregular. Notice widening of the intercellular spaces $($ star $)(\mathbf{E M}, \times \mathbf{8 0 0 0})$. (b) An electron micrograph of a section of group III, showing columnar cells of the basal layer of the corneal epithelium with nuclei $(\mathrm{N})$ and prominent nucleolus (nu). Areas with narrow intercellular spaces (star) are observed with intact desmosomes (circle) in other areas. They lie on a regular basement membrane (arrow) with hemidesmosomes $(\mathbf{E M}, \times \mathbf{8 0 0 0})$. (c) An electron micrograph of a section of group III, showing a nearly normal architecture of the corneal stroma (S) and appearing of keratocytes (K) in between with elongated $(\mathrm{N})$, rough endoplasmic reticulum (arrow) in cytoplasm and small cytoplasmic process (head arrow) (EM, $\times$ 8000). (d) An electron micrograph of a section of group III, showing endothelial cells (arrow) with elongated nucleus (N) and vacuolated cytoplasm (v), these cells rest on moderate electron dense Descemet's membrane (D). Notice lysis in some areas of stroma $(\mathrm{s})(\mathbf{E M}, \times \mathbf{8 0 0 0})$. 
Group IV: it shows nearly normal superficial squamous cell with superficial microvilli. The nucleus has normal configuration. They are connected by electron-dense desmosomes

(Fig.7a).

The basal layer has tall columnar cells with euchromatic nuclei resting on regular basement membrane joining with it by hemidesmosomes (Fig.7b).
Corneal stroma has apparently regular collagen fibers and normal spindle shaped keratocyte with euchromatic nucleus inbetween fibers (Fig.7c).

Descemet's membrane is homogenous electron-dense membrane lined with a single layer of flat endothelial cell that has moderate electron-dense elongated nucleus, mitochondria and pinocytotic vesicles

(Fig.7d).

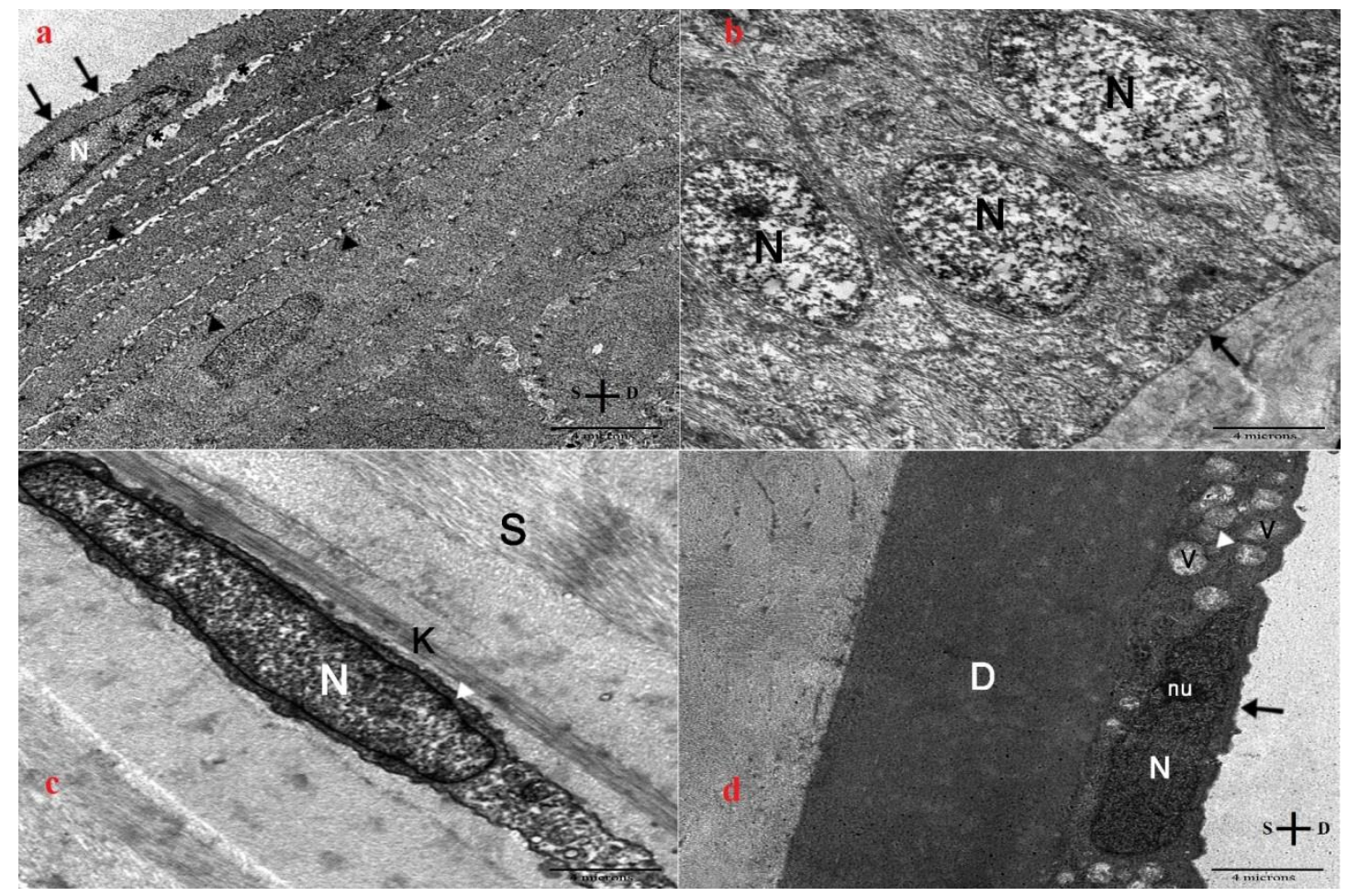

Fig. 7: (a) An electron micrograph of subgroup IV, showing flat cells on the top of the corneal epithelium with normal nuclear $(\mathrm{N})$ configuration and apical microvilli (arrow). Notice appearance of electron-dense desmosomes connecting epithelial cells (head arrow) with very narrow intercellular spaces (star) (EM, $\times \mathbf{8 0 0 0})$. (b) An electron micrograph of group IV, showing tall columnar cells of the basal layer of the corneal epithelium with nearly normal nucleus (N). Regular basement membrane (arrow) and electron-dense spots of hemidesmosomes are observed (EM, $\times$ 8000). (c) An electron micrograph of group IV, showing regular arrangement of the collagen fibers of the corneal stroma (S) and elongated keratocyte $(\mathrm{K})$ showing elongated euchromatic nucleus $(\mathrm{N})$ with scanty cytoplasm (head arrow) (EM, $\times$ 8000). (d) An electron micrograph of group IV, showing electron dense Descemet's membrane (D) and endothelial cells (arrow) with electron dense nucleus $(\mathrm{N})$, prominent nucleolus $(\mathrm{nu})$, mitochondria (head arrow) and pinocytotic vesicles $(\mathrm{V})(\mathbf{E M}, \times \mathbf{8 0 0 0})$. 


\section{Immunohistochemical study:}

Sections of control rat cornea have shown negative reaction for Anti-CD44 in both epithelial and stromal cells (Fig.8a). While positive cells within the stroma and epithelium are detected in Sections of group IV. It is suggested that the MSCs can differentiate into epithelial cells and keratocytes (Fig.8b).

Weak positive immune reaction for vimentin is detected in Sections of group $\mathrm{I}$ and subgroup IIa (Fig.8c \& 8d). While corneal sections of subgroup IIb show moderate positive reaction for vimentin in the stroma which has indicated regeneration and proliferation of cells (Fig.8e).

Sections of groups III and IV stained by vimentin have shown strong positive cytoplasmic reaction in stromal cells which means more regeneration and proliferation of cells (Fig.8f \& 8g).

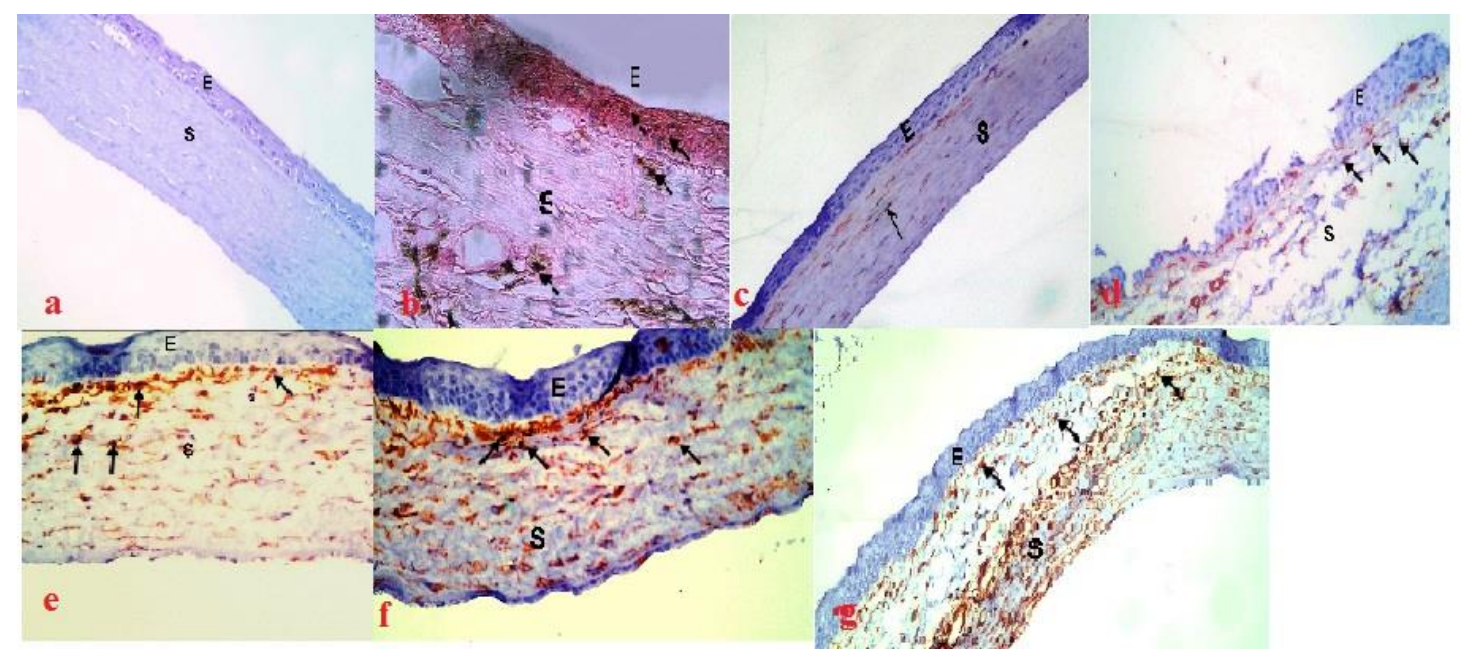

Fig. 8: (a) A photomicrograph of a section of Group I, Showing negative immune-reaction for CD44 i.e. (No brown pigmentation) in the cytoplasm of epithelial (E) and stromal cells (S) (Anti-CD44, $\times$ 400). (b) A photomicrograph of a section of group IV, showing positive expression (brown pigmentation) of CD44 (arrows) in the cytoplasm of stromal cells $(\mathrm{S})$ and in between epithelial cells $(\mathrm{E})($ Anti-CD44, $\times$ 400). (c) A photomicrograph of Group I, Showing weak positive immune-reaction i.e. (brown cytoplasmic staining) $(\uparrow$ ) for vimentin in few stromal cells (s) (vimentin, $\times$ 400). (d). A photomicrograph of a section subgroup IIa, Showing weak positive immune-reaction ( $\uparrow$ ) for vimentin in few stromal cells $(s)$ (vimentin, $\times$ 400). (e) A photomicrograph of a section of subgroup IIb, Showing moderate positive immune-reaction $(\uparrow)$ for vimentin in few stromal cells (s) (vimentin, $\times \mathbf{4 0 0}$ ). (f) A photomicrograph of a section of group III, Showing strong positive immune-reaction $(\uparrow)$ for vimentin in few stromal cells (s) (vimentin, $\times \mathbf{4 0 0 )}$. (g) A photomicrograph of a section of group IV, Showing strong positive immune-reaction i.e. (brown cytoplasmic staining) $(\uparrow)$ for vimentin in few stromal cells (s) (vimentin, $\times$ 400). 


\section{Morphometric and statistical results:}

The mean and SD of corneal epithelial, stroma and Descemet's membrane thicknesses for all groups were represented in Table 1.

In group II, the mean value of the epithelial, stromal and Descemet's membrane thicknesses showed a highly significant differences $(\mathrm{P}<0.01)$ comparing with control group. In group III, the mean value of corneal epithelial and stromal thicknesses showed a highly significant changes $(\mathrm{P}<0.01)$ comparing with control group. The mean value of corneal Descemet's membrane thickness showed non-significant changes comparing with control group.

In group IV, the mean value of corneal epithelial, stroma and Descemet's membrane thicknesses showed non-significant difference compared to the control group.

Table 1: Showing the mean and \pm SD of corneal epithelial, stroma and Descemet's membrane thicknesses ( $\mu \mathrm{m})$ for all groups with comparison between all groups by Post Hoc LSD test.

\begin{tabular}{lllllllllll}
\hline & \multicolumn{1}{c}{ Group I } & \multicolumn{4}{c}{ subGroup IIb } & Group III & \multicolumn{3}{c}{ Group IV } \\
\cline { 2 - 10 } & & $\begin{array}{l}\text { Mean } \pm \text { SD } \\
(\boldsymbol{\mu m})\end{array}$ & $\begin{array}{l}\text { sig. } \\
\mathbf{P}<\mathbf{0 . 0 1}\end{array}$ & $\begin{array}{l}\text { Mean } \pm \text { SD } \\
(\boldsymbol{\mu m})\end{array}$ & $\begin{array}{l}\text { sig. at } \\
\mathbf{P}<\mathbf{0 . 0 1}\end{array}$ & $\begin{array}{l}\text { Mean } \pm \text { SD } \\
(\boldsymbol{\mu m})\end{array}$ & $\begin{array}{l}\text { sig. at } \\
\mathbf{P}<\mathbf{0 . 0 1}\end{array}$ & $\begin{array}{l}\text { Mean } \pm \text { SD } \\
(\boldsymbol{\mu m})\end{array}$ & $\begin{array}{l}\text { sig. } \\
\mathbf{P}<\mathbf{0 . 0 1}\end{array}$ \\
\hline $\begin{array}{l}\text { Thickness } \\
\begin{array}{l}\text { Epithelium } \\
\text { Thickness } \\
\text { stroma }\end{array}\end{array}$ & of & $33.75 \pm 2.76$ & $\mathrm{~b}, \mathrm{c}$ & $13 \pm 1.3$ & $\mathrm{a}, \mathrm{c}, \mathrm{d}$ & $21 \pm 2.5$ & $\mathrm{a}, \mathrm{b}, \mathrm{d}$ & $30.5 \pm 1.1$ & $\mathrm{~b}, \mathrm{c}$ \\
$\begin{array}{l}\text { Thickness } \\
\begin{array}{l}\text { Descemet's } \\
\text { membrane }\end{array}\end{array}$ & of & $52.75 \pm 17.3$ & $\mathrm{~b}, \mathrm{c}, \mathrm{d}$ & $199.75 \pm 16.4$ & $\mathrm{a}, \mathrm{c}, \mathrm{d}$ & $183.5 \pm 12.61$ & $\mathrm{a}, \mathrm{b}, \mathrm{d}$ & $101.25 \pm 7.5$ & $\mathrm{~b}, \mathrm{c}$ \\
\hline
\end{tabular}

$\mathrm{a}=$ Significant with group I $\quad$ b=Significant with subgroup IIb

$\mathrm{c}=$ Significant with group III $\quad \mathrm{d}=$ Significant with group IV

\section{Discussion}

A chemical burn of cornea is one the dangerous ocular injuries resulting in visual impairment. Therefore, it is necessary to use proper management during accurate duration. In this work, corneal sections for subgroup IIa showed that alkali burn induced corneal injury with desquamation of surface epithelium and disturbance with spaces in between collagen fibers of stroma. Ultrastructure observations showed marked disfigurement in their appearance and heterochromatic nuclei. Stroma showed degenerated keratocytes. The endothelial cell 
over it had vacuolated cytoplasm. These results are in agreement with other investigators ${ }^{(2,9)}$ who observed desquamation and degeneration of superficial cells, with regularly arranged collagen fibers in some parts of the stroma but other parts showed separated and disturbed collagen fibers.

Other investigators ${ }^{(10)}$ stated that the guinea pig corneal sections two days after alkali burn, revealed that the epithelial cells and Bowman's membrane were destroyed, leaving necrotic debris with regular collagen bundles in the stroma.

In the present study, examination of corneal sections of subgroup IIb showed variability in thickness of the corneal epithelium with areas of focal thinning. The epithelial cells were disorganized and some showed vacuolated cytoplasm. The stromal fibers showed wide spaces in between and congested blood vessels. Descemet's membrane was detected with an interrupted layer of endothelial cells. However the cells of the superficial layer had flat euchromatic nucleus with mononuclear cell infiltrations.

These results are similar to the previous reports $(2,9,10,11)$ which showed marked ultrastructural changes in the form of separation of the superficial cells from underlying cells and showed multiples cytoplasmic vacuoles with wide intercellular spaces with disfigurement in their shapes and degenerated nuclei, while stroma showed an irregularly arranged collagen fibers with degenerated and neovascularization. The endothelial cell showed cytoplasmic vacuoles.

Some researchers $(10,12)$ found that new corneal blood vessels were variable in size and shape as evidenced by both light and electron microscopy two weeks after alkali burn. This blood vessel had the loosest endothelial junctions that participated in inflammatory process and exchange of cells.

The new blood vessels can induce leakage of plasma and increasing corneal edema which in turn reduces cornea oxygenation and delays wound healing. ${ }^{(13)}$

Alkali burns induced ulcers were accompanied with inflammatory cells infiltration that damaged the normal corneal architecture due to liberation of matrix metalloproteinases and several proteolytic enzymes ${ }^{(14,15,16)}$

In the current study, rats of group III showed organized epithelium and disturbed collagen fibers in anterior part of the stroma. The electrondense desmosomes appeared connecting cells together with narrow intercellular gap between them without inflammatory cell infiltrations. The keratocytes had thin cytoplasmic processes. The endothelial cell was still degenerated. 
Many studies ${ }^{(2,17,18)}$ explained that rats treated by HA for two weeks had a significantly enhanced rate of epithelial defect healing with a multilayered epithelium, morphological regularity, and intercellular adhesion in epithelial cells compared with untreated eyes.

In the present study, alkali burn treated with MSC group showed homing of PKH26 labeled MSCs to the site of injury in cornea which corroborates the reports ${ }^{(9,19,20,21)}$ on MSCs permeation after systemic and other reports ${ }^{(22,23)}$ on MSCs permeation after subconjunctival administration.

In this work, the corneal sections of group IV showed nearly uniform thickness with very thin intact Bowman's. Normal nuclear configuration in the corneal epithelial cells was observed. They were connected by electron dense desmosomes. There were long normal keratocytes in between regular collagen fibers. The endothelial cell appeared normal.

Those results agreed with the results of other investigators $(9,11,20,24)$ who provided evidence that intravenous or topical application of BMMSCs could be used to reconstruct corneal surfaces under light and electron microscopes. Some investigators (25) added that MSCs could differentiate into corneal epithelial cells in vitro.
MSCs enhance corneal epithelial and stromal wound healing after alkali burns injuries through their anti-inflammatory effects and modulating immunity $(22,26,27,28)$.

In the current study, immunohistochemical examination of corneal section using AntiCD44 in control group showed absence of positive cells. This was in agreement with some researchers $(20,29)$ who reported that CD44 positive cells were absent in corneal epithelium. Another researchers (30, 31) detected positive CD44 expression in normal cornea and during corneal epithelial wound healing.

In this work, corneal sections of group IV using Anti-CD44 showed positive cells in epithelium and stroma that was agreed with previous works ${ }^{(9,20,31)}$ showing CD44 positive cells in groups treated with MSCs.

Some reports (32, 33, 34) revealed CD44 expression is one of the characteristics of MSCs in both humans and mice.

In the current work, Immunohistochemical staining of alkali burn group showed relatively increased immune-reaction for vimetnin compared to those of control group which showed faint brown cytoplasmic reaction. The vimentin immune-reaction was more expressed in group III and group IV. 
These findings were in agreement with previous work ${ }^{(20)}$ which reported that rabbits treated with BM-MSCs after corneal alkali burn showed a significant increase in vimentin expression compared to control group and pathological group.

Also, other studies ${ }^{(35,36)}$ showed high levels of vimentin expressed in keratocytes at alkaliinjured stroma.

Other researchers ${ }^{(37)}$ observed high levels of vimentin in the corneal stroma after MSCs transplantation confirming the differentiating ability of MSCs. the MSC population shares many properties of keratocytes and expresses myofibroblastic cell markers a-SMA and vimentin ${ }^{(38)}$

These results have been supported by the statistical results. The mean value of corneal epithelial, stroma and Descemet's membrane thicknesses of group II showed highly significant difference comparing with control group in agreement with results of other work $(2,11)$

Corneal alkali burn caused increasing of the thickness of the cornea due to edema, neovascularization, and inflammatory cell infiltration in the stroma and exudation attached to the endothelium ${ }^{(39) .}$

The mean value of corneal epithelial and stroma thicknesses of group III showed highly significant changes comparing with control group. Those statistical results were disagreed with another researcher ${ }^{(2)}$ who showed HAtreated corneas had a non-significant change in these thicknesses compared with the control group.

In group IV, the mean value of three thicknesses showed non-significant changes comparing with control group in agreement with results of other researchers. ${ }^{(11,20,40)}$ It was due to restoration of corneal keratocytes and endothelial cells by MSCs. ${ }^{(41)}$

Our results demonstrate that the use of MSCs early in corneal chemical trauma is quicker and better healing of the wounded cornea than use of HA.

\section{Conflict of interest:}

None of the contributors declared any conflict of interest.

\section{References}

1. Namrata Sharma, Manpreet Kaur, Tushar Agarwal, Virender S. Sangwan, Rasik B. Vajpayee and Franzcoc (2018) :Treatment of acute ocular chemical burns. Survey of ophthalmology; 63:214-216.

2. Nagwa Kostandy Kalleny and Nevine Bahaa E. Soliman (2011): Light and electron microscopic study on the effect of topically applied hyaluronic acid on experimentally induced corneal alkali burn in albino rats. The Egyptian Journal of Histology, 34: 829-848. 
3. Monika Bieniasz, Andrzej Chmura and Artur Kwiatkowsk (2014): Stem Cells - general characteristic and sources. MEDtube Science Jun;. 2(2):1-9.

4. Ting-Shuai Jiang, Li Cai, Wei-Ying Ji, YanNian Hui, Yu-Sheng Wang, Dan $\mathrm{Hu}$ et al., (2010): Reconstruction of the corneal epithelium with induced marrow mesenchymal stem cells in rats. Molecular Vision; 16:13041316.

5. Abdel Aziz M.T., Wassef M.A., RasheedL.A., Mhfouz S., Omar N., Elsebaie M.M. (2011): Mesenchymal stem cells therapy in acute renal failure: possible role of hepatocyte growth factor. J Stem Cell Res Ther, 1:109-115.

6. Bancroft JD and Layton C (2013): The hematoxylin and eosin, connective and mesenchymal tissues with their stains. In: Suvarna SK, Layton C, Bancroft JD (eds.) Bancroft's theory and practice of histological techniques. 7th Edn. Philadelphia: Churchill Livingstone, 173-212.

7. Hayat MA (2000): Chemical fixation. In: Hayat MA. (ed.) Principles and techniques of electron microscopy: biological applications. 4th Edn. Edinburg, UK: Cambridge University Press, pp. 4-85.

8. Yang MC, Chi NH, Chou NK (2010): The influence of rat mesenchymal stem cell CD44 surface markers on cell growth, fibronectin expression, and cardiomyogenic differentiation on silk fibroin Hyaluronic acid cardiac patches. Biomaterials 31: 854-862.

9. Eman Mohammed Faruk, Aisha El Mansy, Amal Mohmmod Al-Shazly and Neama Mahmoud Taha (2017): Light and Electron Microscopic Study of the Anti-Inflammatory Role of Mesenchymal Stem Cell Therapy in Restoring Corneal Alkali Injury in Adult
Albino Rats. J. stem cell Bio. Transplant; 1:18.

10. Shehab Hafez Mohamed (2011): Light and electron microscopic study of the corneal stroma during the healing process of alkaliinduced ulcer. Egypt J Histol 35:67-73

11. Abeer M El-Mahalaway, Nahla El-Eraky ElAzab, Mohamed Abdrabbo, Omar M Said, Dina Sabry and Mohamed M El Sibaie (2018): Comparative Light and Electron Microscopic Study on the Therapeutic Efficacy of Adipose Derived Stem Cells Versus Exosomes for Experimentally Induced Acute Corneal Injuries in Rats. Stem Cell Res Ther 8: 431-443.

12. Ye J, Yao $\mathrm{K}$ and Kim JC. (2006): Mesenchymal stem cell transplantation in a rabbit corneal alkali burn model: Engraftment and involvement in wound healing. Eye; 20:482-490.

13. Baish JW, Netti PA and Jain RK (1997): Transmural coupling of fluid flow innmicrocirculatory network and interstitium in tumors. Microvasc Res; 53:128-141.

14. Kim DW, Lee SH and Shin MJ (2015): PEP-1FK506BP inhibits alkali burn-induced corneal inflammation on the rat model of corneal alkali injury. BMB Rep; 48:618-623.

15. Bermudez ML, Lago S and Eiro N (2015): Corneal epithelial wound healing and bactericidal effect of conditioned medium from human uterine cervical stem cells. Invest Ophthalmol Vis Sci; 56: 983-992.

16. El-Fattah El-Shazly AA and Ahmed AI (2016): Therapeutic effects of extracts from spirulina platensis versus bevacizumab on inflammationassociated corneal neovascularization. J Med Surg Pathol; 1: 1-7.

17. Stiebel Kalish H, Gaton DD, Weinberger D, Loya N, Schwartz Ventik M and Solomon A (1998): A comparison of the effect of 
hyaluronic acid versus gentamicin on corneal epithelial healing. Eye; 12:829-833.

18. Burgalassi S, Nicosia N, Monti D, Falcone G, Boldrini E, Fabiani O, et al.(2011): Arabinogalactan as active compound in the management of corneal wounds: in vitro toxicity and in vivo investigations on rabbits. Curr Eye Res; 36:21-28.

19. Jiang TS, Cai L, Ji WY, Hui YN, Wang YS, $\mathrm{Hu} \mathrm{D}$ et al., (2010): Reconstruction of the corneal epithelium with induced marrow mesenchymal stem cells in rats. Mol Vis; 16:1304-1316.

20. Soheir Kamal Ahmed, Amel Ali Soliman, Sahar M. M. Omar, Wafaa Rabee Mohammed (2015): Bone Marrow Mesenchymal Stem Cell Transplantation in a Rabbit Corneal Alkali Burn Model (A Histological and Immune Histo-chemical Study). International Journal of Stem Cells; 8(1):1-9.

21. Cejka C, HolanV and Trosan P (2016): The favorable effect of mesenchymal stem cell treatment on the antioxidant protective mechanism in the corneal epithelium and renewal of corneal optical properties changed after alkali burns. Oxid Med Cell Longev; 112.

22. Lin Yao, Zhan-rong Li, Wen-ru Su, Yong-ping Li, Miao-li Lin, Wen-xin Zhang et al., (2012): Role of Mesenchymal Stem Cells on Cornea Wound Healing Induced by Acute Alkali Burn. PLoS ONE 7(2):1-7.

23. Diamantis Almaliotis, Georgios Koliakos, Eleni Papakonstantinou, Anastasia Komnenou, Angelos Thomas, Spiros Petrakis et al., (2015): Mesenchymal stem cells improve healing of the cornea after alkali injury. Graefes Arch Clin Exp Ophthalmol; 253:1121-1135

24. Liu H, Zhang J, Liu CY, Hayashi Y and Kao WW (2012): Bone marrow mesenchymal stem cells can differentiate and assume corneal keratocyte phenotype. J Cell Mol Med; 16:1114-1124.

25. Ting-Shuai Jiang, Li Cai, Wei-Ying Ji, YanNian Hui, Yu-Sheng Wang, Dan Hu, et al., (2010): Reconstruction of the corneal epithelium with induced marrow mesenchymal stem cells in rats. Molecular Vision; 16:13041316.

26. Zeppieri M, Brusini P, Parodi PB (2015): Corneal treatment with adipose derived stem cells. Glo Adv Res J Med Med Sci; 4: 296-300.

27. Frese L, Dijkman PE, Hoerstrup SP (2016): Adipose tissue-derived stem cells in regenerative medicine. Transfus Med Hemother; 43: 268-274.

28. Ghazaryan E, Zhang Y, He Y (2016): Mesenchymal stem cells in corneal neovascularization: comparison of different application routes. Mol Med Rep; 14: $3104-$ 3112.

29. Alho AM and Underhill CB. (1989): The hyaluronate receptor is preferentially expressed on proliferating epithelial cells. J Cell Biol;108:1557-1565

30. Su-Ning Zhu, Bernhard Nölle and Gernot Duncker (1997): Expression of adhesion molecule CD44 on human corneas. British Journal of Ophthalmology; 81:80-84.

31. Soheir A. Filobbos, Dalia H. Abd EL-Aziz, Mohammed H.A. Mostafa and Mai A.M. ELMotasem (2016): Effect of subconjunctival injection of mesenchymal stem cells on alkaliinduced acute corneal injury in rats. Med $\mathbf{J}$ Cairo Univ 84: 303-310.

32. Omayma Helal, Aisha El-Mansy and Wael Abo El-khair (2010): Role of Stem Cells in Regeneration of Myocardium in Experimentally Induced Myocardial Infarction. Egypt J. Histol.; 33(1): 8 -16. 
33. Noha M. Afifia and Olfat N. Reyad (2013): Role of mesenchymal stem cell therapy in restoring ovarian function in a rat model of chemotherapy-induced ovarian failure: a histological and immunohistochemical study. Egypt J Histol 36:114-126.

34. Gao Y., Ruan B., Liu W., Wang J., Yang X., Zhang Z., et 1., (2015): Knockdown of CD44 inhibits the invasion and metastasis of hepatocellular carcinoma both in vitro and in vivo by reversing epithelial-mesenchymal transition. Oncotarget.: 6 (10): 7828- 7837.

35. Wang T and Shi W. (2009): Expression of fibroblast activation proteins in corneal stromal neovascularization. Curr Eye Res; 34:112-117.

36. Lakimenko $\mathrm{S}$, Buznyk $\mathrm{O}$, Shchypun $\mathrm{S}$, Dumbrova N and Molchanyuk N. (2010): Healing of alkali burned rabbit corneas with persistent superficial ulceration after excimer laser phototherapeutic keratectomy. Clinical and electron microscopic findings. Klin Oczna; 112:187-194.

37. Ishizaki M, Zhu G, Haseba T, Shafer SS and Kao WW. (1993): Expression of collagen I, smooth muscle alpha-actin, and vimentin during the healing of alkali-burned and lacerated corneas. Invest Ophthalmol Vis Sci; 34:3320-3328.

38. Heindl LM, Schlötzer-Schrehardt U, Cursiefen C, Bachmann BO, Hofmann-Rummelt $\mathrm{C}$ and Kruse FE. (2011): Myofibroblast metaplasia after descemet membrane endothelial keratoplasty. Am J Ophthalmol; 151:10191023.

39. Liu $X$, Lin Z, Zhou T, Zong R, He H, Liu Z, et al. (2011): Anti-angiogenic and antiinflammatory effects of SERPINAK on corneal injury. PLoS One 2011; 16:1114-1124

40. Saadettin Sel, Ulf M. Schilling, Norbert Nass, Andreas Simm, Fabian Garreis, Matthias Knak, et al., (2012): Bone marrow cells and CD117positive haematopoietic stem cells promote corneal wound healing. Acta Ophthalmol.; 90: 367-373.

41. Coulson-Thomas VJ, Caterson B, Kao WW. (2013): Transplantation of human umbilical mesenchymal stem cells cures the corneal defects of mucopolysaccharidosis VII mice. Stem Cells; 31:2116-2226.

To cite this article: Saadia A. Shalaby, Essam M. Eid, Ali M. Ali, Samia M. Manawy, Samar F. Gad. Comparison between the Effect of Hyaluronic Acid and Mesenchymal Stem Cell Therapy on Cornea of Adult Albino Rats after Exposure to Alkali Burn (Light and Electron Microscopic Study). BMFJ 2020;37 (3):524-541 , DOI: 10.21608/bmfj.2020.20695.1197 